\title{
Evidence for a unique cue in positive patterning
}

\author{
PETER C. HOLLAND and HARVEY BLOCK \\ University of Pittsburgh, Pittsburgh, Pennsylvania
}

\begin{abstract}
Rats received Pavlovian positive patterning training in which a simultaneous tone + light compound was reinforced with food and the light and tone elements were separately nonreinforced. Solution of the discrimination was accompanied by the emergence of a unique response topography in the presence of the compound. Control rats that received reinforced compound presentations but no separate nonreinforced presentations of the elements acquired behaviors characteristic of light and tone stimuli separately paired with food, but did not acquire the unique response topography acquired by the rats that received positive patterning discrimination training. These data supported earlier claims that the solution of such discriminations involves the conditioning of a stimulus unique to the compound stimulus.
\end{abstract}

Most theories of learning assume that the associative strength of a compound stimulus is equal to the summed associative strengths of its constituent elements. This simple assumption has permitted the successful prediction of a number of common conditioning phenomena such as summation, blocking, overshadowing, and conditioned inhibition.

However, the results of a variety of experiments show that under some circumstances animals may treat a stimulus compound and its constituent elements very differently. For example, animals can be taught to respond to a consistently reinforced compound stimulus (AB) but to withhold responding to the separately nonreinforced elements (A and $B$ ) of that compound, or to respond to the separately reinforced elements $A$ and $B$ but to withhold responding to the nonreinforced compound AB (e.g., Forbes \& Holland, 1980; Rescorla, 1972; Woodbury, 1943). Similarly, there have been several recent reports (e.g., Forbes, 1981; Gillette \& Bellingham, 1982) that extended nondifferential experience with stimulus compounds may also result in progressive behavioral differentiation of the compound and its separate elements. All of these findings seem inconsistent with the simple summation notion.

One approach to reconciling these types of data with a simple summation notion is to assume the existence of a compound-specific or unique stimulus in addition to the explicit stimulus elements. That is, an $\mathrm{AB}$ compound could be described as comprising three cues, A, B, and a cue unique to their combination. Rescorla $(1972,1973)$ noted that such an approach, coupled with the power of recent conditioning theories, allows us to account for much of the

This research was supported in part by U.S. Public Health Service Grant MH31396. Reprints are not available. The authors' mailing address is: Department of Psychology, University of Pittsburgh, Pittsburgh, Pennsylvania 15260. available data that seem inconsistent with a simple summation notion. In a series of experiments in which the relative validities of the explicit stimulus elements and of the implicit unique cue were manipulated, Rescorla (1973) found considerable evidence that the properties of such implicit cues paralleled those of explicit stimuli. For example, compound conditioning phenomena like summation, "overexpectation," and "superconditioning" (Rescorla \& Wagner, 1972) that involve both explicit stimulus elements and the implicit unique cue were produced by manipulating the strength of the implicit unique cue.

The present experiment sought another, quite different demonstration of the existence of such a unique cue in stimulus compounds. It made use of Holland's $(1977,1980)$ observation that the form of rats' Pavlovian appetitively conditioned responses (CRs) is greatly influenced by the nature of the conditioned stimulus (CS). For instance, localizable visual cues evoked "rear" and "magazine" behaviors but auditory CSs evoked "head-jerk" and "startle" behaviors (see below for descriptions of those behaviors.) If the constituent elements of a compound were chosen so that they evoked very different CR forms, then perhaps a cue unique to the compound would produce a unique response topography when conditioned.

Rats were trained on a Pavlovian positive patterning $(\mathrm{AB}+, \mathrm{A}-, \mathrm{B}-)$ discrimination in which a tone + light compound was reinforced with food pellets but presentations of the light and tone alone were not reinforced. Presumably, the unique compound cue would acquire considerable associative strength in this discrimination, since it is the most valid predictor of reinforcement (Rescorla, 1972, 1973). Thus, mastery of this discrimination might be expected to involve the acquisition of a unique response topography to the compound, while light- and tonespecific responding to those elements decreased. 
Comparison groups received equivalent reinforced experience with the tone + light compound, but did not receive experience with the individual elements. Thus, the individual elements in those groups were as predictive of reinforcement as was the unique cue, and might be expected to overshadow conditioning to that cue, especially if the unique cue were assumed to be of relatively low salience (Rescorla, 1972, 1973). Consequently, responding to the compound in the comparison groups might be anticipated to reflect simply the combined forms of responding evoked by the visual and auditory stimuli separately.

\section{METHOD}

\section{Subjects and Apparatus}

The subjects were 24 male Sprague-Dawley-strain-derived rats. They were about 120 days old at the beginning of the experiment and were maintained at $80 \%$ of their free-feeding weights throughout the experiment by limiting their access to food. The rats were housed in individual cages with water freely available. The colony room was continuously illuminated.

Four experimental chambers, each $22.9 \times 20.3 \times 20.3 \mathrm{~cm}$, were used. The two end walls of each chamber were aluminum, and the side walls and top were clear acrylic. A food cup was recessed in the center of one end wall. Six centimeters above the food cup was a 6-W lamp covered by a 3.5 -cm-diam half-spherical red lens that protruded $3 \mathrm{~cm}$ into the chamber; illumination of this lamp served as the visual CS in these experiments. The chamber floors were made of $.48-\mathrm{cm}$ stainless steel rods spaced $1.9 \mathrm{~cm}$ apart. Each experimental chamber was enclosed in a soundresistant shell that contained a speaker for delivering the auditory stimulus. The front wall of each shell contained an acrylic window to permit behavioral observations. A low-light television camera was mounted $2.1 \mathrm{~m}$ from the experimental chambers so that all four chambers were included in its field of view. General illumination of the chambers was provided by an infrared lamp mounted behind and above the television camera. A videocassette recorder was programmed to record behaviors that occurred during and 10-sec before and after CS presentations.

\section{Behavioral Observation Procedures}

All observations were made from videotapes. Each rat's behavior was observed at 1.25 -sec intervals during the 10 -sec period immediately prior to CS presentations and during the CS presentations. The observations were paced by auditory signals recorded on the videotapes. On each observation, one, and only one, behavior was recorded. In addition, the occurrence of a startle response was noted for each rat on each trial. Five behavioral categories were reported. Rear-standing on hindlegs with both front feet off the floor. Magazine-standing motionless in front of the food magazine with head or nose within the recessed food cup. Head jerk-short, rapid horizontal and/or vertical movements of the head; all four feet on grid floor. Usually (but not necessarily) directed toward the side of the chamber that contained the food cup. Rear head jerk-simultaneous performance of rear and head-jerk behaviors, i.e., head jerk while rearing on hind legs. Startle-sudden, rapid body movement resulting in movement of all four legs; generally occurred only at onset of auditory stimuli.

\section{Procedure}

All rats first received a single 12 - to 20 -min session in which they were trained to eat from the recessed food cup. Food pellets (45 mg, P. J. Noyes Co.) were presented on a variable-time (VT) 2-min schedule until eight reinforcements had been delivered. The reinforcing event throughout the experiment was the delivery of two food pellets in a .5 -sec interval. All rats then received 18 80 -min sessions. The eight rats in Group $P$ received two 10 -sec reinforced presentations of the compound CS (illumination of the panel lamp + 82-db SPL, $1500-\mathrm{Hz}$ tone) and two nonreinforced presentations of each of the two separate elements. The six stimuli in each session were intermixed randomly with intertrial intervals averaging 9 min each; stimulus order was changed every other session. Two control groups received the same number of reinforced compound trials in each session that Group $P$ received, but received no element-alone presentations. Group $\mathrm{Cl}$ received only the two reinforced compound trials, but Group $\mathrm{C} 2$ received two nonreinforced compound trials as well, thus receiving the same number of reinforced and nonreinforced presentations of the light and tone as Group $P$ received. Intertrial intervals averaged $27 \mathrm{~min}$ in Group $\mathrm{C1}$ and $16 \mathrm{~min}$ in Group C2.

\section{RESULTS AND DISCUSSION}

Figure 1 shows the acquisition of the various behaviors during compound stimulus presentations in all three groups and during the presentation of the individual elements in Group P. All rats acquired startle, head-jerk, magazine, and rear behaviors to the tone + light compound. As training progressed, 6 of the 8 rats in Group P also acquired rear/headjerk behavior during compound presentations. This behavior pattern, in which the subject showed the head movements characteristic of head-jerk behavior while rearing, was always directed toward the visual CS source. Only 3 of the 16 rats (total) in the two control groups acquired this behavior pattern (Mann-Whitney Us $=9$ and 10, respectively, for the comparisons of Group P with Group C1 and Group C2 over the last four sessions). Instead, rats that received reinforced compound presentations without contemporaneous nonreinforced element presentations (Groups $\mathrm{C1}$ and C2) performed both rear and headjerk behaviors during compound presentations, but not simultaneously.

Furthermore, the rats in Group $P$ learned to respond differentially to the compound and its elements: Over the last four sessions, those rats showed significantly more rear/head-jerk and startle behaviors during the tone + light compound than during either the light or the tone separately (Wilcoxon Ts $\leqslant 2$ ). Similarly, head-jerk behavior was numerically more frequent during the compound than during either the tone or the light presented separately, although only the compound versus light difference was reliable $(T=4)$. Conversely, rear behavior in Group $P$ was more frequent $(T=2)$ during the nonreinforced light alone than during the tone + light compound. It is likely that this lower level of rear behavior to the compound was due to competition from other behaviors (startle, head jerk, and rear/head jerk) evoked by the compound that were not evoked by the light alone. Discriminative performance was not evident in performance of magazine behavior (Ts > 11). Finally, there was no evidence of differential responding to the compound and to the element that characteristically evokes a particular behavior (e.g., differential startle behavior between the compound 

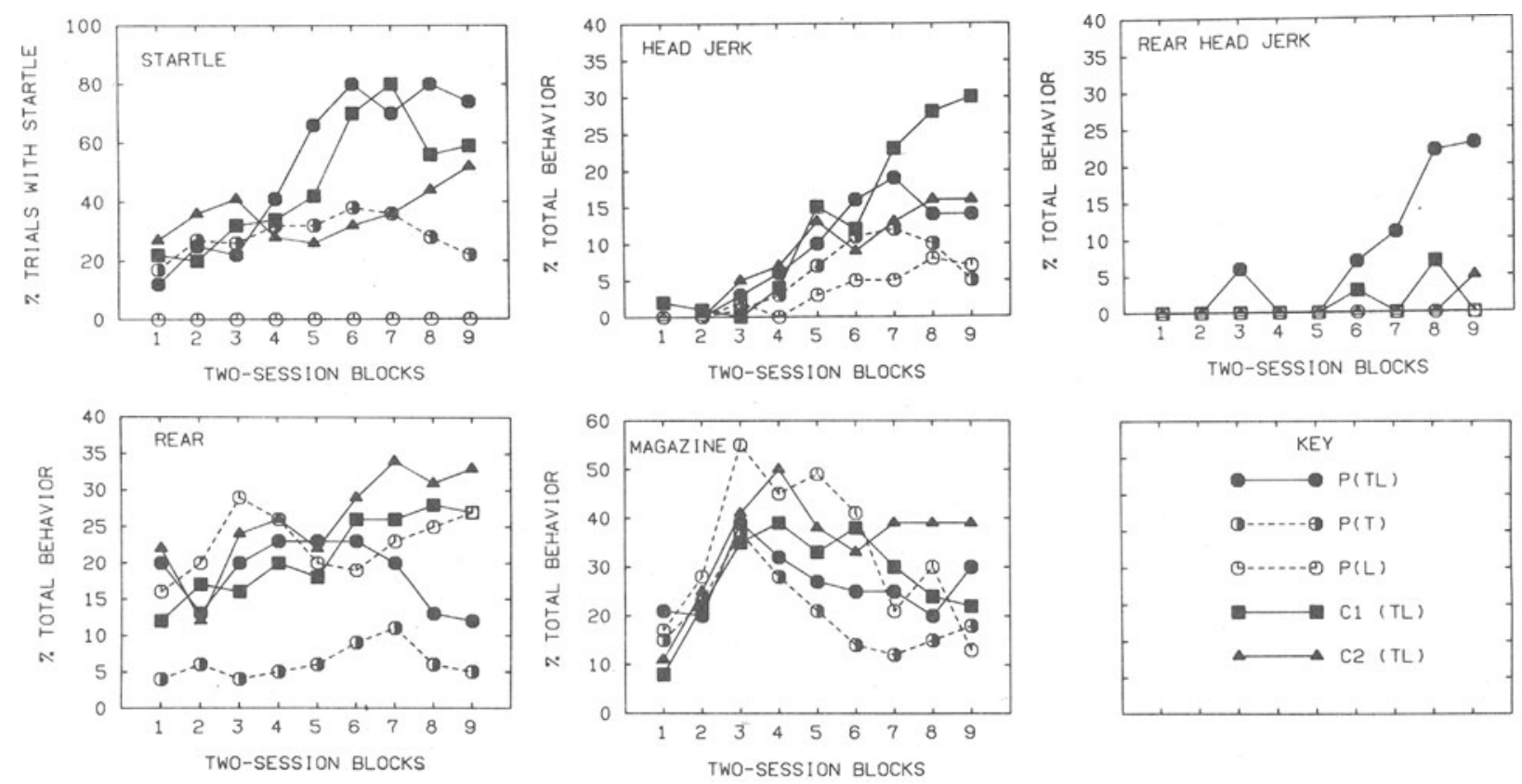

Figure 1. Responding in the presence of the tone + light compound (TL), the tone alone (T), and the light alone (L) in Group P, and to the tone + light compound in Groups $\mathrm{C} 1$ and $\mathrm{C} 2$.

and the tone) until the emergence of the unique response, rear/head jerk.

In addition, there were several differences in the behavior of the two control groups, all of which differences were consistent with previous data on the effects of partial reinforcement (Holland, 1979). Group $\mathrm{C1}$, which received two consistently reinforced presentations of the tone + light compound each day, exhibited reliably more startle $(U=12)$ and head-jerk $(U=12)$ behavior and marginally $(p<.10)$ less rear $(U=17.5)$ and magazine $(U=16)$ behaviors than Group C2, which received two reinforced and two nonreinforced compound presentations each day. Thus, Holland's (1979) data were extended to a situation in which partial and consistent reinforcement groups were matched in numbers of reinforced trials rather than in total numbers of trials (as in the previous experiments.)

The gradual acquisition of a behavior unique to the compound in Group P but not in either of the control groups and the lack of discriminative performance until that behavior emerged is consistent with the notion that simultaneous positive patterning discriminations are solved by the acquisition of associative strength to a low-salience cue unique to the stimulus compound. Rescorla (1972) suggested that association with that cue in simple compound conditioning is normally overshadowed by conditioning to the much more salient explicit stimulus elements, but the separate nonreinforcement of those elements in the positive patterning discrimination makes the unique cue the best predictor of reinforcement. Thus, such training would result in the gradual acquisition of responding to the unique cue as responding to the explicit elements was decremented by nonreinforcement, as was observed here.

It is of interest to compare the present data with those of Ross and Holland (1982), who examined the acquisition of positive patterning using serial (i.e., light-then-tone) compound stimuli in this conditioning preparation. Their subjects showed no evidence of acquiring a response topography unique to the stimulus compound. Instead, the first element (light) "set the occasion" for responding to the subsequent tone. That is, although little conditioned behavior occurred during the light within the stimulus compound, the tone presented immediately after the light evoked substantial tone-specific behavior (head-jerk and startle behaviors). Little conditioned behavior occurred during either the light or tone presented outside the serial compound stimulus.

There is little evidence that subjects in Group $P$ of the present experiment used such an "occasionsetting" strategy in solving the simultaneous positive patterning discrimination. Rear, head-jerk, and magazine behaviors were not reliably more frequent to the compound than to the element that alone generally evoked any one of those behaviors, as would be anticipated if one (or both) of the elements set the occasion for responding to the other element. Perhaps the discriminative performance of startle behavior suggests that the light somewhat set the oc- 
casion for tone responding. But equally likely is the possibility that the suspected unique compound cue itself evoked that response. Supporting that possibility is the observation that startle responding did not occur more frequently to the compound than to the tone alone until the unique behavior rear/head jerk emerged.

\section{REFERENCES}

Forbes, D. T. Spontaneous configuring in conditioned flavor aversion. Unpublished doctoral thesis, University of Pittsburgh, 1981.

Forbes, D. T., \& Holland, P. C. Positive and negative patterning after CS preexposure in flavor aversion conditioning. $A n$ imal Learning \& Behavior, 1980, 8, 595-600.

GilletTe, K., \& Bellingham, W. P. Loss of within-compound flavour associations: Configural preconditioning. Experimental Animal Behaviour, 1982, 1, 1-17.

Holland, P. C. Conditioned stimulus as a determinant of the form of the Pavlovian conditioned response. Journal of Experimental Psychology: Animal Behavior Processes, 1977, 3, 77-104.

Holland, P. C. Differential effects of omission contingencies on various components of Pavlovian appetitive conditioned behavior in rats. Journal of Experimental Psychology: Animal Behavior Processes, 1979, 5, 178-193.

Holland, P. C. Influence of visual conditioned stimulus characteristics on the form of Pavlovian appetitive conditioned responding in rats. Journal of Experimental Psychology: Animal Behavior Processes, 1980, 6, 81-97.

RESCORLA, R. A. "Configural" conditioning in discrete-trial bar pressing. Journal of Comparative and Physiological Psychology, 1972, 79, 307-317.

Rescorla, R. A. Evidence for a "unique stimulus" account of configural conditioning. Journal of Comparative and Physiological Psychology, 1973, 85, 331-338.

Rescorla, R. A., \& Wagner, A. R. A theory of Pavlovian conditioning: Variations in the effectiveness of reinforcement and nonreinforcement. In A. H. Black \& W. F. Prokasy (Eds.), Classical conditioning II. New York: Appleton-Century-Crofts, 1972.

Ross, R. T., \& Holland, P. C. Serial positive patterning: Implications for "occasion-setting." Bulletin of the Psychonomic Society, 1982, 19, 159-162.

WoodBury, C. B. The learning of stimulus patterns by dogs. Journal of Comparative Psychology, 1943, 35, 29-40.

(Manuscript received for publication May 19, 1983.) 